\title{
Possible anti-psoriasis effect of the methanol extract of Phoenix dactylifera L. seeds
}

\author{
Zeena A Hussein $^{*{ }^{\circledR}}$, Nada S Shaker $^{1}$, Nibras J Tahseen ${ }^{\circledR}$, Ahmed M Al-Tuhafi $^{\circledR}{ }^{\circledR}$ Ahmed F Mutee $^{1^{\circledR}}$ \\ ${ }^{1}$ Pharmacy Department, Al-Rasheed University College, Baghdad, Iraq
}

\section{A R T I C L E I N F O}

Article Type:

Original Article

\section{Article History:}

Received: 1 April 2020

Accepted: 19 June 2020

\section{Keywords:}

Psoriasis

Imiquimod

Inflammatory score

Angiogenesis

Phoenix seeds

\begin{abstract}
A B S T R A C T
Introduction: Psoriasis is an autoimmune, chronic and debilitating disorder, mainly characterized by the presence of inflamed, dry and scaly patches of skin. This study aimed to investigate the possible anti-psoriasis effect of methanolic extract of Phoenix dactylifera seeds in mice.

Methods: Thirty-five Wistar albino male mice were divided randomly into 5 groups $(\mathrm{n}=7)$. Group 1 represented the normal group (received the cream base only), group 2 through 5 received a single daily application of imiquimod 5\% cream; while, groups 3, 4 and 5 received once daily application of clobetasol $0.05 \%$, P. dactylifera seeds methanol extract $2 \%$ and $5 \%$, respectively. All animals received the test substances on the shaved back for 10 consecutive days. Scoring for skin inflammation severity (scaling, erythema and thickness) was recorded on daily basis, and the animals were sacrificed on day 11. Skin and spleen samples were taken to evaluate histopathological and spleen index changes, respectively.

Results: Imiquimod successfully induced psoriasis like lesion in mice as well as significant increase in the skin neovascularization. Topical methanol extract of $P$. dactylifera cream significantly reduced the signs of inflammation as well as skin neovascularization in a concentration-dependent manner, where 5\% methanol extract cream showed better reduction in the inflammatory parameters than $2 \%$ methanol extract cream. These findings were supported by the results of the histopathological examination.

Conclusion: Results of the study suggest that methanol extract of $P$. dactylifera seeds possesses anti-psoriasis activity and could be used efficiently for the alleviation of psoriatic symptoms.
\end{abstract}

Implication for health policy/practice/research/medical education:

Methanol extract of Phoenix dactylifera seeds showed good anti-psoriasis activity by reducing inflammatory signs and excessive skin vascularization. Hence, it might be considered as a beneficial therapeutic alternative for psoriasis patients.

Please cite this paper as: Hussein ZA, Shaker NS, Tahseen NJ, Al-Tuhafi AM, Mutee AF. Possible anti-psoriasis effect of the methanol extract of Phoenix dactylifera L. seeds. J Herbmed Pharmacol. 2020;9(4):382-390. doi: 10.34172/jhp.2020.48.

\section{Introduction}

Psoriasis is a chronic autoimmune disorder with appearance of abnormal skin patches. These patches are usually red, dry, itchy and scaly. There are five main types of psoriasis: plaque, guttate, inverse, pustular, and erythrodermic. Severity of psoriasis ranging from mild, small, localized patches to complete body coverage. This disease is chronic, and characterized by recurrent exacerbations that are emotionally and physically debilitating (1). Psoriasis is generally thought to be a genetic disease that is triggered by environmental factors. Symptoms often worsen during winter and with certain medications, such as beta blockers or non-steroidal antiinflammatory drugs (2). Infections and psychological stress can also play a role (1). The interaction between hyper-proliferative keratinocytes, inflammatory dendritic cells, neutrophils, mast cells, and $\mathrm{T}$ cells, induces the development of psoriatic lesions. IL-23 and IL-17 are considered the main inflammatory mediators produced by different cell types, including $\mathrm{T}$ cells, neutrophils, natural killer cells, natural killer $\mathrm{T}$ cells, and mast cells which have all been found the main contributors to the pathogenesis in psoriasis (3). Angiogenesis appears to be a first-order event in psoriatic arthritis, as in rheumatoid 
arthritis. Alterations in the vascular morphology of the nail folds of patients affected by psoriasis can be seen by microscopic examination. Also, an increase in the number of blood vessels and morphological vascular alterations, such as increased branching and elongated blood vessels, have been observed in the psoriasis synovial membrane (4). Treatments of psoriasis include a range of topical and systemic therapies as well as phototherapy. Medications may also be prescribed to reduce pain and disability from arthritis and other manifestations. Treatment is based on psoriasis severity. Mild psoriasis usually is treated with topical therapy (corticosteroids, vitamin D analogues and others) and phototherapy in the case of insufficient response. Systemic therapy should be considered in case of moderate to severe psoriasis. Commonly used systemic medications are methotrexate, ciclosporin, acitretin and etretinate; however, sometime biological agents like fumaric acid esters are also used (5).

Imiquimod is an immune modulator which is available commercially as $5 \%$ cream. Topical imiquimod stimulates the innate and adaptive immune responses and promotes cytokine production. This has given rise for being used in the treatment of different types of benign and malignant skin conditions due to its potential antiviral, antitumor, and immune-regulatory effects. Imiquimod is approved by the Food and Drug Administration (FDA) for the treatment of anogenital warts, actinic keratosis, and superficial basal cell carcinomas (6). Imiquimod is a ligand for the toll-like receptors TLR7 and TLR8. It exacerbates psoriasis at both local treated areas and distant sites. Hence, it has led to the development of animal research models of psoriasis. Application of Aldara cream on the ears and back of mice results in the development of psoriasis-like lesions within 5 days of application and is explained by an increased influx of various inflammatory cells as well as hyperplasia of the epidermis (7).

Phoenix dactylifera $\mathrm{L}$. is also known as date palm. It is an important member of the family Arecaceae. The date palm is considered as one of the most important sources of food for human. Traditionally, the fruit is used for a sore throat, cold, relief of fever, abdominal problems, while the pollens are used by the Egyptians to improve fertility in women. The seeds of date palm are used in animal feed to improve growth; the oil of the seeds is used in cosmetics, and the quality of these products is found to be encouraging (8).

The whole plant contains various constituents ranging from vitamins, minerals, and proteins to phytochemicals including phenolic compounds and phenolic acids, flavonoids, sterols, anthocyanins, carbohydrates and fibers (9). Date palm seeds were found to be a good source of fatty acids, phenolic acids, and flavonoids, sterols, dietary fibers, proteins, minerals, vitamins and antioxidants. The seeds of $P$. dactylifera are interesting for research in the field of psoriasis due to previous studies supporting that the seeds possess anti-angiogenesis, anti-inflammatory as well as anti-oxidant activities (8). This study aims to investigate the possible anti-psoriatic effect of methanolic extract of $P$. dactylifera L. seeds in mice and elucidate the possible underlying mechanism of its therapeutic effect.

\section{Materials and Methods}

Materials

Seeds of $P$. dactylifera L. species (Khestawa) were obtained from Iraqi Date Factory, Baghdad, Iraq and identified for authenticity by Department of Pharmacognosy, College of Pharmacy, Al-Mustansiriyah University. Imiquimod cream was obtained commercially as (Aldara $5 \%$ ) cream by MEDA Pharmaceuticals (Sweden). Clobetasol cream was obtained commercially as (Dermovet $0.05 \%$ ) cream by GSK Pharmaceuticals (UK). Hydrophilic cream base (Aqueous cream) was obtained commercially by Xpel marketing (UK).

\section{Extraction process}

Date seeds were cleansed from the remaining fruit flesh and washed with tap water, then left to dry. Olive seeds were then oven dried at $40^{\circ} \mathrm{C}$ for 30 minutes and left to cool down, then grounded into a fine powder using a heavyduty stainless-steel grinder. The final weight obtained from the powder was 200 g. P. dactylifera seeds powder was extracted with absolute methanol solvent; using the cold method (maceration) for the extraction. The powder was soaked with the solvent at a ratio of 1:4 (W/V) and left in a shaking water bath at $40{ }^{\circ} \mathrm{C}$ for 24 hours, then filtered in the next day using Whatman no. 1 filter paper; this step was repeated three times. The filtrate was collected and then concentrated using the rotary evaporator (Buchi, Switzerland). The concentrated extract was then put in a freeze dryer to obtain the final lyophilized powder of the extract. The yield percentage for the extract was then calculated (10).

Preparation of the extract topical cream

A topical cream was prepared from the methanolic crude extract of $P$. dactylifera seeds. Two concentrations were prepared from $P$. dactylifera seeds methanol extract $(2 \%$ PDS-ME w/w and 5\% PDS-ME w/w), by weighing $2 \mathrm{~g}$ and $5 \mathrm{~g}$ respectively of the crude extract and mixing them first with a small amount of glycerin (about $1 \mathrm{ml}$ ) to achieve homogenization. Then, it was incorporated gradually to the hydrophilic cream base to get the final weight (100 g). The hydrophilic cream base was purchased commercially and served as the vehicle; its main constituent being "liquid paraffin, glyceryl stearate, petrolatum, stearyl alcohol, glycerin, butylene glycol, sodium benzoate, carbomer, triethanolamine and water" (11).

Experimental animals

The study was conducted in physiology lab, Pharmacy Department, Al-Rasheed University College after 
obtaining ethical committee approval. The experimental procedures were followed National Institute of Health Guidelines for the care and use of laboratory animals and the European Council Directive on 24 November 1986 for Care and Use of Laboratory Animals (86/609/EEC). Thirty-five Wistar albino male mice aging 6-8 weeks old with an average weight of $27 \pm 3$ g were randomly divided into 5 groups $(n=7)$. The mice were housed under specific pathogen-free conditions with $23 \pm 2^{\circ} \mathrm{C}$ temperature and 12 hours light/night cycle, and left to acclimatize for at least one week. Animals were fed with formal forage and water ad libitum in the animal house of Pharmacy Department, Al-Rasheed University College. An area of $5 \mathrm{~cm} \times 4 \mathrm{~cm}$ was shaved from the backs of mice and the test samples were applied once daily on the shaved back for 10 days. On day 1 and continuing for 10 consecutive days, mice in all groups except for the normal group received a daily topical dose of $62.5 \mathrm{mg}$ of the IMQ cream on the shaved area of the dorsal skin $(12,13)$. group 1 (normal) received a topical hydrophilic cream base only; group 2 (induced) received a topical IMQ only; group 3 received $0.05 \%$ clobetasol cream and IMQ (imiquimod) concomitantly; and groups $4 \& 5$ received a topical cream preparation with two concentrations of methanol extract of $P$. dactylifera L. seeds (PDS-ME 2\%) and (PDS-ME 5\%), respectively along with IMQ for 10 continuous days on the shaved area of the dorsal skin. The entire experimental design is summarized in Figure 1.
Scoring of skin inflammation severity

Signs and severity of inflammation were observed and recorded according to a scoring system developed based on the clinical Psoriasis Area and Severity Index (PASI). Erythema, scales and skin thickness were scored independently on a scale from 0 to 4 ; where $0=$ none; 1 = slight; $2=$ moderate; $3=$ marked and $4=$ very marked. The cumulative score (erythema plus scaling plus skin thickness) was served to indicate the severity of inflammation (scale 0-12) (14).

\section{Sample collection}

At the end of the experiment (day 11), the animals were put under general anesthesia with diethyl ether and sacrificed by cervical dislocation, then skin tissues and spleen were collected for further investigations.

\section{Quantification of microscopic vascularization}

The dorsal skin and adjacent soft tissue of the mice were excised and photographed from the reverse side. The number of vascular branching and elongation per dorsal skin sample was counted (15).

\section{Changes of spleen in mice}

Each mouse was weighed before being sacrificed, after scarification, each spleen was excised and weighed. Spleen index was calculated (spleen weight/ body weight) to reflect the dynamic body changes of the mice (16).

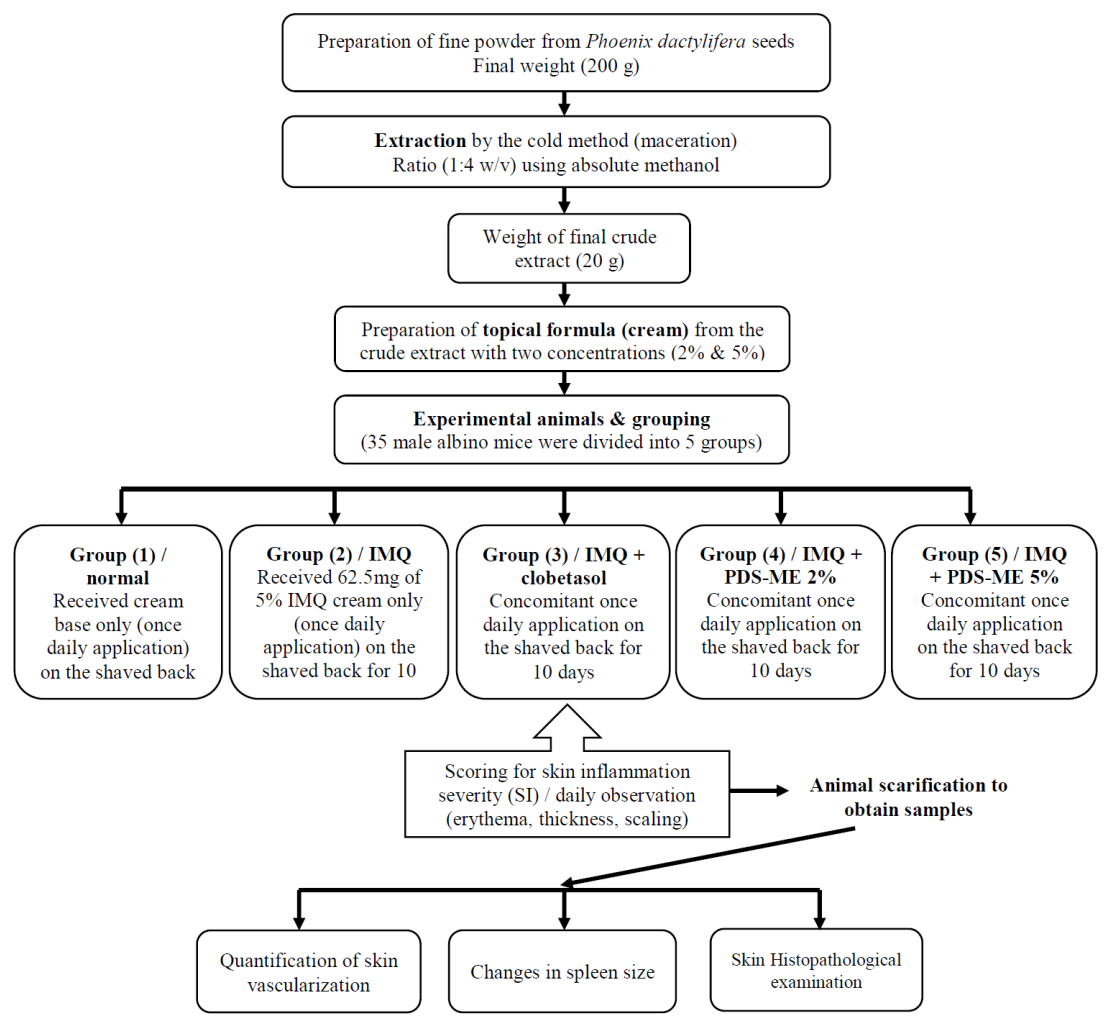

Figure 1. Schematic illustration of the experimental design. IMQ, Imiquimod; PDS-ME, Phoenix dactylifera seeds methanol extract; SI, severity of inflammation. 


\section{Histopathological examination}

Skin samples from back lesions of each mouse were fixed in $10 \%$ formaldehyde for $48-72$ hours and then were processed to obtain the final tissue - paraffin embedded blocks. Sections (thickness, $5 \mu \mathrm{m}$ ) were stained with hematoxylin and eosin (H\&E) and tissue pathology was observed under a light microscope (16).

\section{Statistical analysis}

The experiment statistical analysis was carried out by using the SPSS version 25.0 and Microsoft Office Excel 2010. The results are presented as means \pm SD (standard deviation) and the difference between groups was compared by one-way ANOVA followed by student $t$ test and considered significant at $P \leq 0.05$, high significant at $P \leq 0.01$, and very high significant at $P \leq 0.001$.

\section{Results}

\section{Extraction process}

The weight and yield percentage of the crude extract was $20 \mathrm{~g}$ and $10 \%$, respectively of $P$. dactylifera seeds powder after extracting $200 \mathrm{~g}$ of the powder with absolute methanol using the maceration method of extraction.

\section{Scoring for skin inflammation severity}

The results of this study revealed that at day 3 of starting the experiment, signs of erythema, scaling and dorsal skin thickness started to appear. Thereafter, the intensity of psoriasis-like symptoms in the IMQ-induced group gradually increased in severity till the end of the experiment (day 10), which indicates successful induction of psoriasis like dermatitis in mice. There was a significant difference between the IMQ-induced group and the normal group mice regarding the psoriasis-like symptoms and the cumulative severity of inflammation $(\mathrm{SI})(P<0.001)$ as shown in Figures 2 and 3. However, normal group which was treated with cream base only, did not exhibit any signs of inflammation on the dorsal skin. It was found that there was a statistically significant reduction in the signs of inflammation (erythema, scaling and skin thickness) as well as the SI in the animal groups treated with PDS-ME $2 \%$ and PDS-ME 5\% when compared to the IMQ-induced group $(P<0.001)$. Also, there was a significant difference between animal group treated with PDS-ME $2 \%$ and PDSME 5\% $(P<0.05)$; where animals treated with PDS-ME $5 \%$ exhibited a better effect in reduction of psoriasis-like symptoms than the animals treated with PDS-ME $2 \%$, which means a concentration dependent effect as shown in Figure 2. Animals treated with clobetasol showed significant reduction and improvement in the symptoms of psoriasis like dermatitis $(P<0.001)$ throughout the duration of the experiment when compared to the IMQinduced group. The inhibitory effect of PDS-ME 5\% regarding the SI was almost close to the effect of clobetasol on the symptoms of psoriasis like dermatitis. At the end of the experiment, PDS-ME treated groups in different concentrations exhibited a significant inhibitory effect on IMQ-induced psoriasis-like dermatitis $(P<0.001)$.

Effect on dorsal skin neovascularization

Mice dorsal skin was excised at the end of the experiment (day 11), and photographed to evaluate the increase or decrease of neovascularization. The application of IMQ daily resulted in a significant increase in the blood vessel branching and formation of small blood vessels $(P<0.001)$, as well as the dilatation of the original blood vessels when compared to normal group. The dorsal skin of clobetasol treated animals showed a statistically significant reduction in the vasculature network, where blood vessels appeared to be retracted and constricted when compared to IMQ-

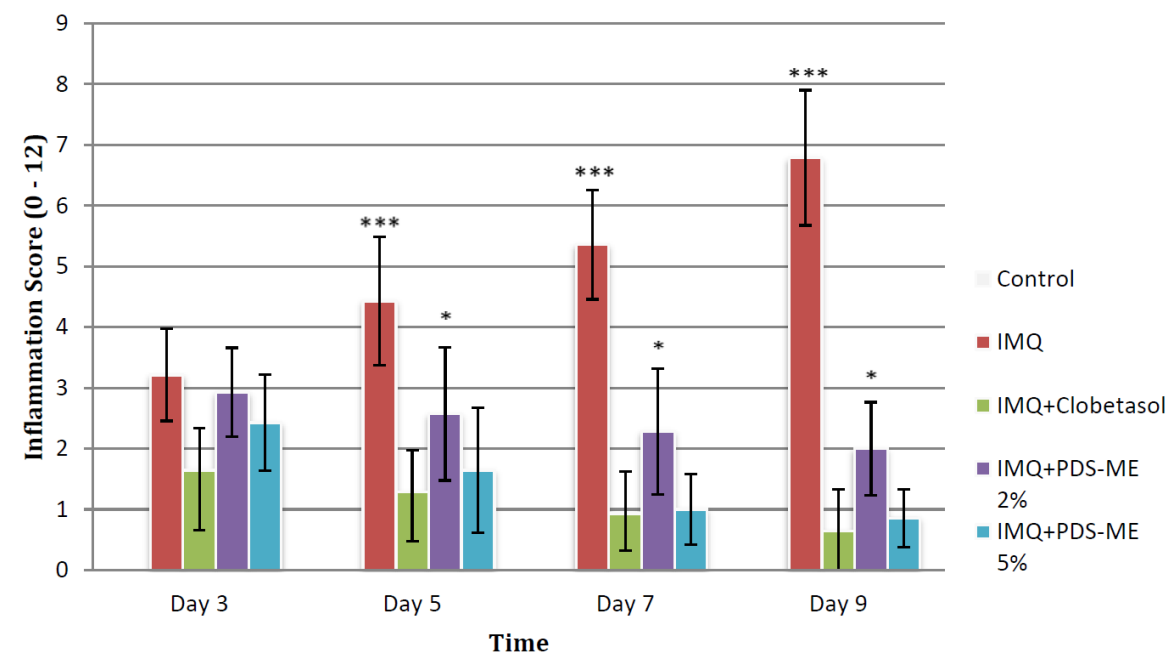

Figure 2. The cumulative score for severity of inflammation ( $\mathrm{SI}$ ) which is the summation of erythema, thickness and scaling for each animal group. Results are presented as mean $+\mathrm{SD},(n=7) .{ }^{*}$ difference between PDS-ME $2 \%$ \& $5 \%(P<0.05) ;{ }^{* * *}$ difference between PDS-ME, clobetasol and IMQ $(P<0.001)$. IMQ, Imiquimod; PDS-ME, Phoenix dactylifera seeds methanol extract. 


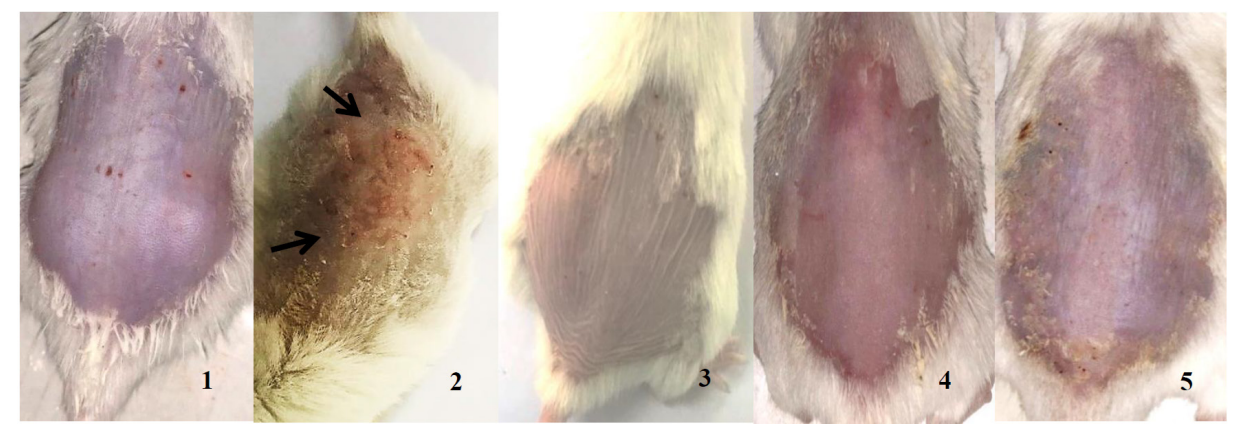

Figure 3. Scoring for skin inflammation severity, the images show various levels of inflammation of the dorsal skin where the test substances were applied on the day 9 of the experiment. (1) represents the normal group, (2) represents IMQ-induced group, (3) represents the group treated with standard clobetasol, (4) represents the group treated with PDS-ME 2\%, and (5) represents the group treated with PDS-ME 5\%. IMQ, Imiquimod; PDS-ME, Phoenix dactylifera seeds methanol extract.

induced group and normal group $(P<0.001)$. PDS-ME $2 \%$ and $5 \%$ treated groups exhibited a significant reduction in blood vessels branching when compared to IMQ-induced group $(P<0.001)$ and the vascular network was almost close to the normal group. PDS-ME 5\% treated animals revealed a better reduction in vascular network when compared to PDS-ME 2\% treated animals, which proves a concentration dependent inhibitory effect on the vascular network of the dorsal skin $(P<0.05)$ as shown in Figures 4 and 5.

Spleen index and splenomegaly

Spleen samples were collected at the end of the experiment (day 11); the size and weight of spleen were assessed for each animal grouping and spleen index was determined. The size and weight of the spleen were markedly enlarged in IMQ-induced psoriasis animals when compared to the normal group. The ratio of spleen weight to body weight was significantly decreased in clobetasol treated group, PDS-ME 2\% treated group and PDS-ME 5\% treated group compared to IMQ-induced group $(P<0.001)$; but not to the levels of ratio of the normal group. Also, there was a statistically significant difference in the reduction of the

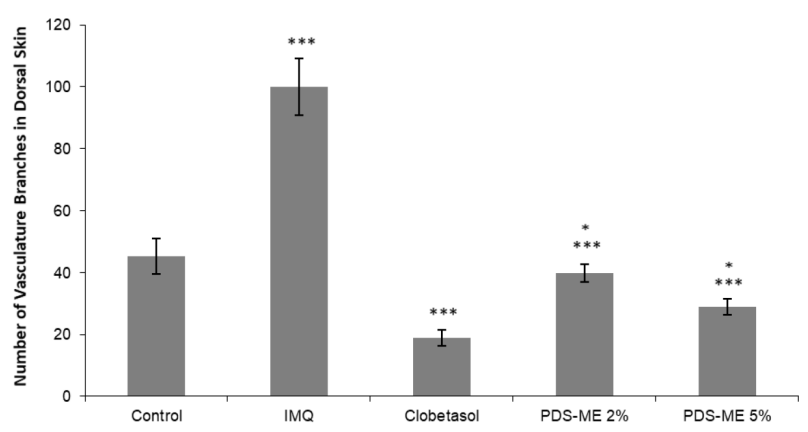

Figure 4. Effect of IMQ, clobetasol and PDS-ME on vasculature network in dorsal skin of mice for each of the animal grouping.

Data are presented as mean $+\mathrm{SD},(n=7)$, * difference between PDSME $2 \%$ \& $5 \%(P<0.05)$, *** difference between PDS-ME, clobetasol and IMQ $(P<0.001)$. IMQ, Imiquimod; PDS-ME, Phoenix dactylifera seeds methanol extract. ratio between PDS-ME $2 \%$ treated animals and PDS-ME $5 \%$ treated animals, where the results were better for the latter $(P<0.05)$ as shown in Figures 6 and 7.

\section{Histological examination}

In animals of group 1 (normal), the histological section showed normal dermal region appearance of stratified epithelium; while the section of the dorsal skin of animals treated with IMQ (group 2) revealed marked hyperkeratosis (black arrows), acanthosis of stratified epithelium (white arrows) with increased rete ridges and abundant inflammatory cells infiltration in the upper dermis layer (yellow arrows) as shown in Figure 8 (panel 2). This supports the results obtained from the gross examination of the dorsal skin (score for skin inflammation severity). Also, the dorsal skin section of animals treated with clobetasol (group 3), showed apparent reduction in thickness of epidermal layer with loss of rete ridges and no visible appearance of inflammatory cells. As for animals treated with PDS-ME, there was a significant reduction in the inflammatory response with varying degrees. The section of skin to animals treated with PDSME 2\% showed that there was still acanthosis of epidermis (black arrows) with rete ridge but to a lesser extent (white arrows) and fewer inflammatory cells in the upper dermis layer, while PDS-ME 5\% treated animals showed better

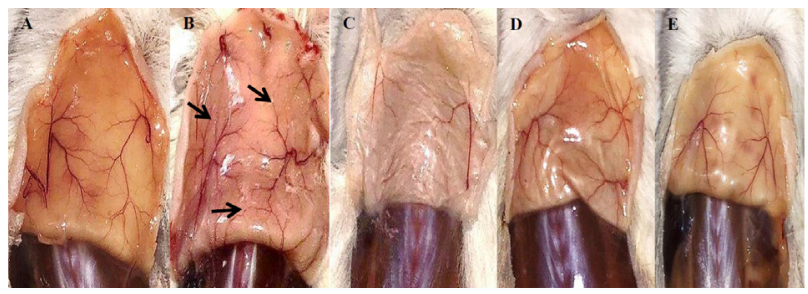

Figure 5. Effect on dorsal skin neovascularization, the images show a various level of angiogenesis of the dorsal skin. (A) represents the normal group, (B) represents IMQ-induced group, (C) represents group treated with standard clobetasol, (D) represents group treated with PDS-ME $2 \%$, and $(E)$ represents group treated with PDS-ME 5\%. IMQ, Imiquimod; PDS-ME, Phoenix dactylifera seeds methanol extract. 


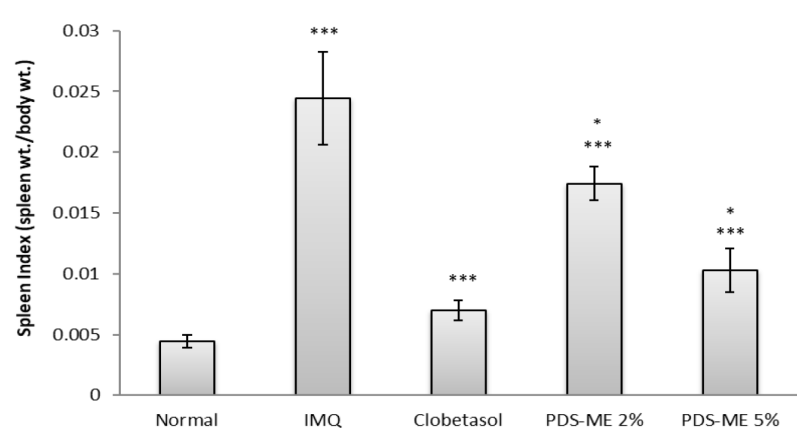

Figure 6. Changes in spleen index (ratio of spleen weight to body weight) in response to test substances for each animal group.

Data are presented as mean $+\mathrm{SD},(\mathrm{n}=7)$, * difference between PDSME $2 \%$ \& $5 \%(P<0.05),{ }^{* * *}$ difference between PDS-ME, clobetasol and IMQ $(P<0.001)$. IMQ, Imiquimod; PDS-ME, Phoenix dactylifera seeds methanol extract.

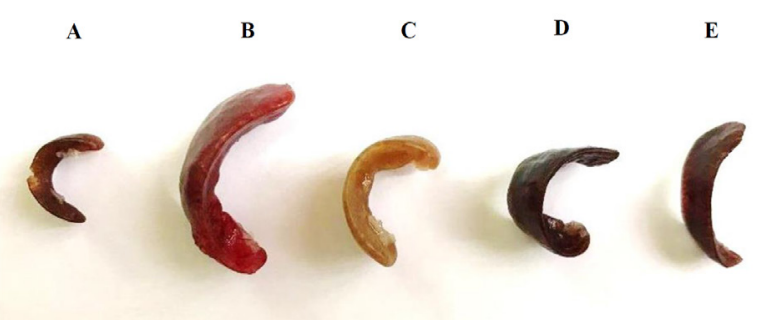

Figure 7. Changes in spleen size and weight in response to test substances for each animal group. (A) represents the normal group, (B) represents IMQ-induced psoriasis group, (C) represents clobetasol treated animals, (D) represents PDS-ME 2\% treated animals and $(E)$ represents PDS-ME 5\% treated animals. IMQ, Imiquimod; PDS-ME, Phoenix dactylifera seeds methanol extract.

results with the section showing the epidermis layer near the normal appearance without rete ridge and no visible inflammatory cells as explained in Figure 8 (panels 4 and 5), respectively.

\section{Discussion}

The selection of methanol as the extraction solvent was based on previous study done in 2016, where P. dactylifera L. seeds powder was extracted with different solvents of varying polarities and methanol extract gave the highest percentage yield and the best activity. Maceration was used as the extraction process because this method is suitable for thermolabile constituents and to ensure that the essential phytochemical constituents won't be subjected to degradation by higher temperatures $(8,17)$. A topical cream was prepared to study therapeutic effect of methanolic crude extract of $P$. dactylifera seeds. Two concentrations were prepared to measure dose-dependent effect of the extract (2\% PDS-ME w/w and 5\% PDS-ME $\mathrm{w} / \mathrm{w})$. Selection of the mentioned concentrations was based on the studies which determined the acute toxicity (LD50) and sub-acute toxicity of $P$. dactylifera seeds
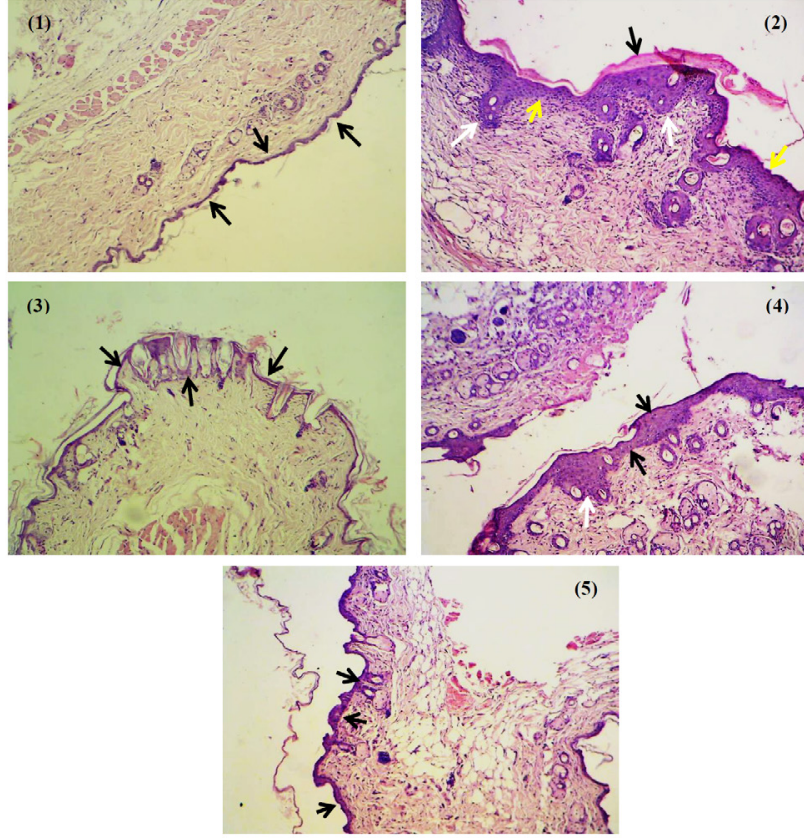

Figure 8. Histological examination of the dorsal skin section. Panel (1) represents normal group, section shows normal structure appearance of dermal region; Panel (2) represents IMQ-induced group, section hyperkeratosis (black arrow), acanthosis of stratified epithelium (white arrow) with increased in rete ridges (yellow arrow) and abundant inflammatory cells infiltration in the upper dermis layer; Panel (3) represents clobetasol treated group, section shows significantly reduced thickness of epidermis layer. Panel (4) represents PDS-ME 2\% group, section shows that still there was acanthosis (black arrow) with rete ridge (white arrow) but to a lesser extent with few inflammatory cells; Panel (5) represents PDS-ME 5\% group, section shows the epidermis layer near the normal appearance $(\times 10),(H \& E)$

aqueous extract; in which, the study demonstrated a dose as high as $5000 \mathrm{mg} / \mathrm{kg}$ did not produce lethal effects for the acute toxicity and a dose as high as $1000 \mathrm{mg} / \mathrm{kg}$ was considered safe for chronic use (18).

All IMQ-treated mice developed characteristic psoriatic signs such as erythema, skin thickening, scaling, and hyperkeratosis (Figure 2). This is because imiquimod is an agonist of the toll-like receptor-7/8 (TLR-7/8) and activation of such receptors can lead to unwanted inflammatory response (19). IMQ-induced psoriasislike inflammation is considered a clean model from immunological viewpoint, resembling most of the features of human psoriatic lesions. It presents epidermal changes based on keratinocyte hyper-proliferation and altered differentiation, also there is increased presence of inflammatory cells including $\mathrm{T}$ cells, dendritic cells, and neutrophils; which can lead to altered vascularity (20). The ability of PDS-ME to reduce the signs of IMQ induced psoriasis like dermatitis could be due to date seeds are considered a wealthy source of phytochemicals like sterols, phenolics, carotenoids, procyanidins, anthocyanins and flavonoids (21). There is a strong relationship between oxidative stress and the pathogenesis of psoriasis, where 
reactive oxygen species (ROS) activate certain signaling pathways in the pathogenesis of psoriasis such as mitogenactivated protein kinase/activator protein 1, nuclear factor kappaB, and Janus kinase-signal transducers (22). Date seeds are known as an important source of flavonoids and hydroxylated derivatives of benzoic acid (gallic acid, protocatechuic acid, p-hydroxybenzoic acid and vanillic acid) and cinnamic acid derivatives (caffeic acid, p-coumaric acid, ferulic acid, m-coumaric and o-coumaric acid), all of which have shown remarkable antioxidant activities both in in vitro and in vivo assays, as well as antiinflammatory effect (23). Thus, antioxidant strategies have proven to be beneficial therapeutics regarding psoriasis. Clobetasol was used as a standard agent in this study, and revealed a significant improvement in the symptoms of psoriasis like dermatitis. This could be explained by the anti-inflammatory and immunosuppressive effect of clobetasol, since it is moderately potent corticosteroid, hence, interfering with the IL-36 and IL-23/IL-17 loop that underlies the pathogenesis of psoriasis (24). Also, a previous study provided pharmacological evidence that topical application of corticosteroids like clobetasol, effectively prevents IMQ-induced psoriasis-like skin inflammation in mice by preventing gene expressions of different types of cytokines related to Th1 cells, Th17 cells and keratinocytes that are known to have essential roles in the pathogenesis of psoriasis (25).

Angiogenesis has been linked to the pathogenesis of psoriasis through the vascular endothelial growth factor (VEGF) and its effect on the vascular network of the skin, and the expression of essential inflammatory mediators (TNF- $\alpha$, IL-17 and IL-23) for the development of psoriasis (26). Daily application of IMQ resulted in the expansion of the skin vascular network (Figure 4). The explanation for this is that IMQ controls the formation of psoriasis inflammatory response through increased expression of IL-17 and IL-23 (pro-inflammatory cytokines) which in return stimulate the expression of VEGF responsible for vasodilatation and angiogenesis (27). Clobetasol belongs to the corticosteroids group, which have vasoconstrictive, anti-proliferative, antiinflammatory and immunosuppressive effect by binding to intracellular corticosteroid receptors. They also regulate gene transcription of numerous genes, particularly those that code for pro-inflammatory cytokines; thus, down regulating the expression of interleukins and TNF- $\alpha$ (28). This could explain the significant reduction and retraction of the dorsal skin vascular network of animals treated with clobetasol. Methanol extract of $P$. dactylifera seeds revealed a significant reduction in skin blood vessels, where 5\% PDS-ME showed better reduction than 2\% PDS-ME (Figures 4 and 5). This is supported by a study in which $P$. dactylifera seeds exhibited an anti-angiogenic effect with methanol extract having the highest percentage of inhibition (8). The extract has been shown to contain polyphenols, flavonoids, proanthocyanidins, cinnamic acid derivatives, catechin and epicatechin as well as ascorbic acid (29). These polyphenols appear to possess various biological effects like antioxidant, anti-mutagenic, anti-carcinogenic, anti-inflammatory and anti-angiogenic activities. Usually by reducing ROS level, the extract either indirectly decreases the expression of pro-inflammatory cytokines and VEGF or directly by affecting different signaling pathways of inflammation and angiogenesis (30); thus, explaining the probable effect of $P$. dactylifera seeds methanol extract on the reduction and improvement of psoriasis like lesion.

Splenomegaly which is a systemic effect of IMQ may result from unintended oral uptake through mice licking their back with IMQ applicated skin (Figure 6). The systemic effect of the methanolic extract of $P$. dactylifera seeds was probably attributed also to the unintended licking of the substance from the back (31).

The inhibitory effect of PDS-ME on induced psoriasis like lesion could be attributed to the phytochemical constituents of $P$. dactylifera; where they can down regulate the expression of different cytokines either by targeting ROS or different signaling pathways in psoriasis process (8). A study revealed the presence of some benzoic acid derivatives like (vanillic acid and cinnamic acid), and some aliphatic alcohols in methanol extract of $P$. dactylifera. These phytochemicals possess a remarkable set of biological activities due to their potent free radical scavenging effects (29). One of the benzoic acid derivatives detected was 5-HMF (5-Hydroxymethyl-2-furfural) and it was investigated for its anticancer properties as well as its anti-inflammatory effect. It was found in a recent study that 5-HMF possessed anti-inflammatory effect by reducing production of nitric oxide, prostaglandin E2 and pro-inflammatory cytokines (TNF- $\alpha$, IL- $1 \beta$ and IL-6) in a concentration-dependent manner as well as downregulating the expression of NF- $\kappa \mathrm{B}$ which contributes greatly in the inflammation cascade (32). This finding supports the previous explained results and the reduced signs of skin inflammation, as well as reduced number of vasculatures in the skin obtained by methanol extract of $P$. dactylifera seeds.

\section{Conclusion}

The anti-psoriatic effect of $P$. dactylifera methanol extract cream was evaluated in IMQ induced psoriasis like dermatitis in a mouse model. The cumulative score of IMQ + PDS-ME 5\% was significantly lower than IMQ + PDS-ME 2\% compared to IMQ group. Due to its approved anti-angiogenic activity, PDS-ME significantly reduced the number of skin vasculature and in a concentration dependent manner with PDS-ME 5\%, which showed higher reduction than PDS-ME 2\%. Spleen index of groups, which received clobetasol, PDS-ME 2\%, and PDS-ME 5\% was lower than that of IMQ group. Hence, 
splenomegaly indicated a possible cellular hyperplasia of the spleen and elevated immune reactions in the body. The effect produced by methanol extract of $P$. dactylifera could be due to the activities of 5-HMF and other phenolic compounds through their direct or indirect effect on the inflammatory cascade of psoriasis. These findings suggest that PDS-ME cream might be used efficiently and safely for the alleviation of psoriatic symptoms.

\section{Acknowledgment}

Authors would like to give thanks to Professor Dr. Salim Rasheed Al-Obaidy, Scientific Deputy Dean in University of Baghdad / College of Medicine for his help in the histopathological part of the experiment, and Assis. Lecturer Mayssam Hussein for her contribution.

\section{Authors' contributions}

ZA and NS did the concept and design of the study, as well as the interpretation of the experimental results. Literature research and results statistical analysis were done by ZA and NJ. Manuscript preparation and editing were done by ZA, AM and AF. All authors read and confirmed the last edition of the manuscript and confirmed for publication.

\section{Conflict of Interest}

All authors declare no conflict of interest regarding this work

\section{Ethical Considerations}

Animal housing and handling, as well as scarification with usage of proper anesthesia and minimal pain infliction were approved by institutional ethical committee of AlRasheed University College / Pharmacy Department; Ref. No.: 2019/PD-006.

\section{Funding/Support}

All the work done in this research was funded by the authors themselves.

\section{References}

1. Parisi R, Symmons DP, Griffiths CE, Ashcroft DM. Global epidemiology of psoriasis: a systematic review of incidence and prevalence. J Investig Dermatol. 2013;133:377-85. doi: 10.1038/jid.2012.339.

2. Kamiya K, Kishimoto M, Sugai J, Komine M, Ohtsuki M. Risk factors for the development of psoriasis. Int J Mol Sci. 2019;20(18):43-47

3. Chiricozzi A, Romanelli P, Volpe E, Borsellino G, Romanelli M. Scanning the immunopathogenesis of psoriasis. Int J Mol Sci. 2018;19(1):E179.

4. Zill JM, Dirmaier J, Augustin M, Dwinger S, Christalle E, Härter M, et al. Psychosocial distress of patients with psoriasis: protocol for an assessment of care needs and the development of a supportive intervention. JMIR Res Protoc. 2018;7(2):e22.

5. Tram NT, Le Son H. Assessment of anti-psoriatic activity of Cassia fistula L. extract incorporated cream. Br J Pharm
Res. 2015;5:370-378.

6. Hanna E, Abadi R, Abbas O. Imiquimod in dermatology: an overview. Int J Dermatol. 2016;55(8):831-844

7. Palomo J, Troccaz S, Talabot-Ayer D, Rodriguez E, Palmer G. The severity of imiquimod-induced mouse skin inflammation is independent of endogenous IL-38 expression. PLoS One. 2018;13(3):e0194667. doi: 10.1371/ journal.pone.0194667.

8. Al-Zubaidy AA, Sahib HB, Hussein ZA. The antiangiogenic activity of Phoenix dactylifera seeds extracts. Int J Pharm Pharm Sci. 2016;8(1):311-315.

9. Deshpande NM and Deshpande MM. Date fruit (Phoenix dactylifera Linn) - a review on nutritional values, phytochemicals and pharmacological actions. World J Pharm Res. 2017;6(8):419-26

10. Dharajiya D, Pagi N, Jasani H, Patel P. Antimicrobial activity and phytochemical screening of Aloe vera (Aloe barbadensis Miller). Int J Curr Microbiol Appl Sci. 2017;6(3):2152-62.

11. Lee SY, Nam S, Kim S, Koo JS, Hong IK, Kim H, et al. Therapeutic efficacies of Artemisia capillaris extract cream formulation in imiquimod-induced psoriasis models. Evid Based Complement Alternat Med. 2018;2018:3610494. doi: $10.1155 / 2018 / 3610494$.

12. Sun J, Zhao Y, and Hu J. Curcumin inhibits imiquimodinduced psoriasis-like inflammation by inhibiting IL-1beta and IL-6 production in mice. PLoS One. 2013;8(6):e67078. doi: 10.1371/journal.pone.0067078

13. Na Takuathung $M$, Wongnoppavich A, Panthong A, et al. Antipsoriatic effects of wannachawee recipe on imiquimodinduced psoriasis-like dermatitis in BALB/c Mice. Evid Based Complement Alternat Med. 2018;2018:7931031. doi: $10.1155 / 2018 / 7931031$

14. van der Fits L, Mourits S, Voerman JSA, Kant M, Boon L, Laman JD, et al. Imiquimod-induced psoriasis-like skin inflammation in mice is mediated via the IL-23/IL-17 axis. J Immunol. 2009;182(9):5836-45.

15. Buerger C, Shirsath N, Lang V, Diehl S, Kaufmann R, Weiger A, et al. Blocking mTOR signalling with rapamycin ameliorates imiquimod-induced psoriasis in mice. Acta Derm Venereol. 2017;97:1087-94.

16. Arora N, Shah K, Pandey-Rai S. Inhibition of imiquimodinduced psoriasis-like dermatitis in mice by herbal extracts from some Indian medicinal plants. Protoplasma. 2016;253(2):503-15. doi: 10.1007/s00709-015-0829-y.

17. Abubakar AR, Haque M. Preparation of medicinal plants: basic extraction and fractionation procedures for experimental purposes. J Pharm Bioall Sci. 2020;12:1-10.

18. Fakhria S, Shokoohinia P, Marami M, Ghiasvand N, Hosseinzadeh L, Shokoohinia Y. Acute and sub-chronic toxicity evaluation of aqueous extract of Phoenix dactylifera seeds in wistar rats, J Rep Pharm Sci. 2018;7(2):319-330.

19. Horváth S, Komlódi R, Perkecz A, Pintér E, Gyulai $\mathrm{R}$, Kemeny A. Methodological refinement of Aldarainduced psoriasiform dermatitis model in mice. Sci Rep. 2019;9(1):3685. doi: 10.1038/s41598-019-39903-x.

20. Lin Y, Yang S, Chen C, Kao H, Fang J, Simon M. Using imiquimod-induced psoriasis-like skin as a model to measure the skin penetration of anti-psoriatic drugs. PLoS One. 2015;10(9):e0137890. doi: 10.1371/journal. pone. 0137890 
21. Anjum FM, Bukhat SI, El-Ghorab AH, Khan MI, Nadeem M, Hussain S, et al. Phytochemical characteristics of Date Palm (Phoenix dactylifera) fruit extracts. Pak J Food Sci. 2012;22(3):117-27.

22. Cannavò SP, Riso G, Casciaro M, Di Salvo E, Gangemi S. Oxidative stress involvement in psoriasis:a systematic review. Free Radic Res. 2019;53(8):829-40.

23. Djaoudene O, López V, Cásedas G, Les F, Schisano C, Bey MB, et al. Phoenix dactylifera L. seeds:a by-product as a source of bioactive compounds with antioxidant and enzyme inhibitory properties. Food Funct. 2019;10:49534965.

24. Boehncke WH, Schön MP. Psoriasis. Lancet. 2015;386:98394. doi: 10.1016/S0140-6736(14)61909-7

25. Mori H, Arita K, Yamaguchi T, Hirai M, and Kurebayashi Y. Effects of topical application of betamethasone on imiquimod-induced psoriasis-like skin inflammation in mice. Kobe J Med Sci. 2016;62(4):79-88.

26. Akhtar T, Wani WY, Kamal MA, Kaur R. Role of angiogenic growth factors in psoriasis:a review. Curr Drug Metab. 2018;19(11):910-6.

27. Wu HH, Xie WL, Zhao YK, Liu JH, Luo DQ. Imiquimod increases cutaneous VEGF expression in imiquimodinduced psoriatic mouse model. Curr Vasc Pharmacol. 2016;14(3):275-9. doi: 10.2174/1570161114666160106151 837.

28. Torsekar R, Gautam MM. Topical therapies in psoriasis. Indian Dermatol Online J. 2017;8(4):235-245.

29. Hussein ZA, Al-Zubaidy AA, Sahib HB. The antiangiogenic activity of Phoenix dactylifera seeds methanol extract in vivo study. Iranian J Pharm Sci. 2018;14(2):83-92.

30. Liu Z, Ren Z, Zhang J, Chuang CC, Kandaswamy E, Zhou T, et al. Role of ROS and nutritional antioxidants in human diseases. Front Physiol. 2018;9:477. doi: 10.3389/ fphys.2018.00477.

31. Grine L, Steeland S, Van Ryckeghem S, Ballegeer M, Lienenklaus S, Weiss S, et al. Topical imiquimod yields systemic effects due to unintended oral uptake. Sci Rep. 2016;6:20134. doi: 10.1038/srep20134.

32. Kong F, Lee BH, Wei K. 5-Hydroxymethylfurfural mitigates lipopolysaccharide-stimulated inflammation via suppression of MAPK, NF- $\mathrm{BB}$ and mTOR activation in RAW 264.7 Cells. Molecules. 2019;24(2):275. 